\title{
Internal Fixation of Proximal Humerus Fracture by PHILOS Plate
}

Hesham Mohamed Safwat, Mohamed Ebrahim Abulsoud, Abdelazim Elsayed Abdelazim

Department of Orthopedic Surgery, Faculty of Medicine, Al-Azhar University, Cairo, Egypt

Corresponding author: Abdelazim Elsayed Abdelazim, Mobile: 00201064498822, email: g01065949954@ gmail.com

\begin{abstract}
Background: Fractures of the proximal humerus are relatively common, accounting for about $5 \%$ to $9 \%$ of all fractions. Majority of undisplaced proximal humeral fractures can be treated with a sling immobilization and physical therapy. However, approximately $20 \%$ of displaced proximal humeral fractures require surgery.

Objective: The aim of the current study was to assess the expected outcomes and complication rate of proximal humerus fractures treated with Proximal Humerus Internal Locking System (PHILOS) plating.

Patients and Methods: This prospective study included a total of 15 patients with fracture of the proximal humerus attending at Department of Orthopedic Surgery, Al-Azhar University Hospitals. This study was conducted between January 2018 to October 2018. All cases were surgically managed by fracture fixation procedures (PHILOS). The follow up period of the cases is 6 months.

Results: From analysis of the literature concerning the use of PHILOS plates, our results were satisfying in terms of functional recovery, with Constant Scores 73.67 that range between (48\%-94\%). Data from our survey show that most of patients were satisfied. We have noticed that limited functional recovery was usually caused by incorrect or delayed rehabilitation. The complication rate in our study $(26.66 \%)$ is comparable to the literature, where the most recent studies report complication rates between 32 and $50 \%$.
\end{abstract}

Conclusion: More accurate length measurement and shorter screw selection should prevent primary screw perforation.

Keywords: Range of motion, PHILOS.

\section{INTRODUCTION}

Fractures of the proximal humerus are relatively common, accounting for about $5 \%$ to $9 \%$ of all fractions ${ }^{(\mathbf{1})}$.

These fractures can be a challenge for orthopedic surgeons in bone therapy because of the nature of osteoporosis generally in the elderly and the relative deformed forces of the surrounding muscles. Fractions are classified according to Neer standards, and treatment is often directed through the relative displacement of anatomical fragments. Historically undeveloped fractures have been treated conservatively, with generally good results ${ }^{(2)}$.

Displaced fractures with angulation of the articular surface $>45^{\circ}$ and displacement of the major segments $>1 \mathrm{~cm}$ have been treated surgically, as have fractures with substantial valgus impaction, all with mixed results ${ }^{(3,4)}$.

Surgical techniques have included percutaneous fixation, standard plate-and-screw fixation, intramedullary fixation with rods or pins, the use of tension bands with and without plates or rods, standard plate modification into blade plate constructs, and hemiarthroplasty ${ }^{(2,4,5)}$.

Proximal humerus fractures are increasingly common in societies with maturing populations. These fractures are not simple to treat. A variety of options exists; however, outcomes are less than ideal in many patients ${ }^{(6)}$.

Most proximal humerus fractures are either non displaced or minimally displaced and can be treated non surgically. Nonsurgical options focus on early functional exercises with the goal of achieving a functionally acceptable range of motion (ROM). For the $15 \%$ to $20 \%$ of displaced proximal humerus fractures that may benefit from surgery ${ }^{(5)}$.

Surgeons should be familiar with the various treatment options available, including recent advances in complex fracture management and lock pad technology, which are particularly important for the care of fractures ${ }^{(7)}$.

Lock pad technology and the use of osteobiologics may become increasingly important in the management of displaced adrenal fractures, making it easier to maintain the humerus head in appropriately selected patients ${ }^{(8)}$.

The aim of the current study was to assess the expected outcomes and complication rate of proximal humerus fractures treated with Proximal Humerus Internal Locking System (PHILOS ) plating.

\section{PATIENTS AND METHODS}

This prospective study included a total of 15 patients with fracture of the proximal humerus attending at Department of Orthopedic Surgery, Al-Azhar University Hospitals.

Approval of the ethical committee and a written informed consent from all the subjects were obtained.

This study was conducted between January 2018 to October 2018. 
All cases were surgically managed by fracture fixation procedures (PHILOS ). The follow up period of the cases is 6 months.

Patients were 9 females (60\%) and 6 males (40\%), aged from 25 to 70 years, with a mean age of 46.86 years. Eight of them had fractures on the right side, while seven patients on the left side. 8 patients had three-part fractures and 7 patients had four-part fractures. The lag time between trauma and surgery ranged from 2 days to a maximum of 7 days, with a mean of 4 days.

Like all other operative procedures in orthopedics, management of fractures of proximal humerus has preoperative, intra-operative and postoperative stages.

Pre-operative Stage: This includes: Patient counselling. Clinical evaluation (history, general examination and local examination). Radiological evaluation. Preoperative preparation of the patient.

Patient Counseling: The followings were discussed with the patients at depth: +The proposed procedure. Preoperative investigations. Details of operative procedure. Post-operative rehabilitation program and average time of this program. The possible complications. The anticipated outcome. Patient counseling was essential in order to decrease patient anxiety and to reach the maximum cooperation of the patient.

Clinical Evaluation: Each patient in this study was carefully assessed clinically by taking a detailed clinical history and performing a thorough examination.

Clinical History: The clinical history was aimed to cover the following: The mechanism of trauma. Any pre-existing history of shoulder injury or dislocation. Hand dominance. Occupation. Any previous surgery, especially involving the shoulder. Pre-assessment of the patient by anesthesiologist to identify fitness for surgery (general history taken about cardiac, chest, renal, and hepatic problems, and chronic medical illness as diabetes mellitus or hypertension).

Clinical Examination: A comprehensive general examination of each patient was performed. After stabilizing the patient's general condition, local clinical examination was performed with particular emphasis on the following:

Complete neurovascular examination of the involved upper extremity: Assessment of peripheral arterial pulsation at the affected extremity was performed to detect any possible insult to any artery. Whenever the patient's condition permitted, a complete neurological examination was performed to assess any brachial plexus injury with special attention to the axillary nerve for light touch and pinprick in its autogenous area over the deltoid.
Deltoid examination: Shoulder contour was inspected for any asymmetry. The patients were asked to abduct the arm against resistance while palpating the deltoid for contraction while carefully observing to detect muscle contraction but it was difficult to be done because of pain. Then, the following demographic data and diagnosis sheet is then completed: Patient research number. Name. Age. Date of trauma. Gender. Patient's activity level.

Patient's job. Affected side. Dominant side. Diagnosis. Neurovascular status. Previous shoulder troubles. Any previous surgical procedure done before. Associated medical illness.

Radiological Evaluation: A precise radiological evaluation is the corner stone for a proper management of fractures of the proximal humerus. The aim of preoperative radiological evaluation was to: Determine the fracture type. Understand the fracture pattern. Detect associated fractures or dislocations. Bone stock of the fragments. Radiological evaluation consisted mainly of plain radiographies and CT scans.

Plain radiography: Each patient underwent a shoulder trauma series which included: True anteroposterior view: This view was obtained by placing the posterior aspect of the affected shoulder up against the X-ray plate and the opposite shoulder was tilted forward approximately 35 degrees. In this view, there is no overlap between the humerus and the glenoid $^{(9)}$.

Scapular Y lateral view: This view was obtained by placing the anterior aspect of the affected shoulder against the X-ray plate, and the other shoulder is tilted forward approximately 35 degrees. The X-ray tube was then placed posteriorly along the scapular spine. The scapular "Y" view is useful in the evaluation of anterior and posterior shoulder dislocations ${ }^{(9)}$.

Velpeau axillary view: With the shoulder sling in place, the patient stood at the end of the $\mathrm{x}$ ray table and leaned backwards 25 to 35 degrees over the table. The $\mathrm{x}$-ray cassette was placed on the table directly beneath the shoulder, and the $\mathrm{x}$-ray machine was placed directly over the shoulder so that the beam passed vertically from superior to inferior, through the shoulder joint onto the cassette ${ }^{(9)}$.

CT scan: CT scanning is an adjunctive method for evaluating complex proximal humeral fractures. When CT scans and standard radiographs were compared, CT scans were superior in assessing the location of fracture lines, displacement of fracture fragments relative to their normal position, rotation of the humeral articular surface, and fractures of both the glenoid and humeral head ${ }^{(10)}$. Although CT scan is not a standard procedure, all our patients had a preoperative CT scan for a better preoperative planning. After acquiring full 
radiological evaluation, the fracture was classified according to Neer classification.

\section{Preoperative Preparation of the Patient:}

Two units of blood were prepared for each patient, but their use was according to the individual situation.

Operative technique: Asepsis: Patients were operated in conventional operating room. The number of persons in the operative theatre was kept to a minimum, as possible. Traffic in and out of the theater was minimized as possible. The skin edges of the wound were sealed from the rest of the wound with plastic sheets.

Surgical Approach: Deltopectoral approach has been used for all the patients.

\section{Surgical techniques}

\section{Implants used: Proximal humeral locked plate.}

Patient Transfer procedure: The transfer procedure is supervised. The arm is held in a pouch arm sling.

Recovery Room: The patient was observed and pulse oximeter was applied to record the pulse and the oxygen saturation. In addition, the blood pressure was checked.

In the Ward: Antibiotics: Patients received intravenous third generation cephalosporin for 4 days postoperatively. Analgesia: Patient controlled analgesia was continued for the first 3648 hours. Investigations and protocol for blood transfusion: Post-operative haemoglobin percent was requested at the second day of surgery and packed red cells were given if haemoglobin percent was $<7 \mathrm{gm} / \mathrm{dl}$. Second-day post-operative radiographs were done. Wound condition: Wound condition was followed during the first 2 weeks and then stitches were removed. Rehabilitation: A wellstructured rehabilitation program can significantly influence the final outcome of the surgery. Patients were well informed about the importance of full compliance to the rehabilitation program with no under or over doing of the exercises. Rehabilitation should begin from the first post-operative day in ascending manner, which can be summoned in three phases.

\section{Statistical Analysis}

Data will be collected, revised, coded and entered to the Statistical Package for Social Science (IBM SPSS) version 24 and the following will be done: Qualitative data will be presented as number and percentages while quantitative data will be presented as mean, standard deviations and ranges. The confidence interval was set to $95 \%$ and the margin of error accepted was set to $5 \%$. So, the pvalue was considered significant as the following: $\mathrm{P}$ $>0.05$ : Non significant. $\mathrm{P}<0.05$ : Significant. $\mathrm{P}<$ 0.01: Highly significant.

\section{RESULTS}

Constant scoring system was used to assess every patient postoperative at 6 months.

Table (1): Descriptive Statistics for age, from injury to operation, FWD flexion, Abduction, Final constant score.

\begin{tabular}{|l|c|c|}
\hline \multirow{2}{*}{ Age (years) } & \multicolumn{2}{|c|}{ Descriptive Statistics } \\
\cline { 2 - 3 } & Range & Mean \pm SD \\
\hline Parts & $25-70$ & $46.87 \pm 16.11$ \\
\hline Flexion & $3-4$ & $3.47 \pm .52$ \\
\hline Abduction & $90-170$ & $139.67 \pm 28.44$ \\
\hline Contra lateral score & $70-160$ & $122.07 \pm 30.12$ \\
\hline Const score & $70-99$ & $86.20 \pm 8.43$ \\
\hline Day operation & $48-94$ & $73.67 \pm 14.01$ \\
\hline
\end{tabular}

Table (2): Relation between Pain score and gender in patients group.

\begin{tabular}{|c|c|c|c|c|c|c|c|c|}
\hline & \multicolumn{6}{|c|}{ Pain } & \multirow{3}{*}{$\chi^{2}$} & \multirow{3}{*}{$\mathbf{P}$} \\
\hline & \multicolumn{2}{|r|}{ no } & \multicolumn{2}{|c|}{ mild } & \multicolumn{2}{|c|}{ moderate } & & \\
\hline & No & $\%$ & No & $\%$ & No & $\%$ & & \\
\hline \multicolumn{9}{|l|}{ Sex } \\
\hline Male & 2 & $33.3 \%$ & 3 & $50.0 \%$ & 1 & $33.3 \%$ & \multirow{2}{*}{0.417} & \multirow{2}{*}{0.812} \\
\hline Female & 4 & $66.7 \%$ & 3 & $50.0 \%$ & 2 & $66.7 \%$ & & \\
\hline
\end{tabular}

Table (2) show that is no significant relation between gender and pain score where $\mathrm{P}$-value $=0.812$.

No pain: 4 cases $(66.67 \%)$ were females and 2 cases $(33.33 \%)$ males which 6 cases in all patients group. 
Internal Fixation of Proximal Humerus Fracture...

Table (3): Relation between Pain score and type of fracture in patients group.

\begin{tabular}{|c|c|c|c|c|c|}
\hline \multirow{2}{*}{\multicolumn{2}{|c|}{ Type of Fracture }} & \multicolumn{4}{|c|}{ Pain } \\
\hline & & No & Mild & Moderate & Total \\
\hline \multirow{2}{*}{ 3part } & $\mathbf{N}$ & 6 & 2 & 0 & 8 \\
\hline & $\%$ & 100.00 & 33.33 & 0.00 & 53.33 \\
\hline \multirow{2}{*}{ 4part } & $\mathbf{N}$ & 0 & 4 & 3 & 7 \\
\hline & $\%$ & 0.00 & 66.67 & 100.00 & 46.67 \\
\hline \multirow{2}{*}{ Total } & $\mathbf{N}$ & 6 & 6 & 3 & 15 \\
\hline & $\%$ & 100.00 & 100.00 & 100.00 & 100.00 \\
\hline \multirow{2}{*}{ Chi-square } & $\mathbf{X}^{2}$ & \multicolumn{4}{|c|}{11.873} \\
\hline & P-value & \multicolumn{4}{|c|}{$0.018^{*}$} \\
\hline
\end{tabular}

Table (3) show that is significant relation between Type of Fracture and pain score where P-value $=0.018^{*}$.

Table (4): Relation between Pain score and fracture side in patients group

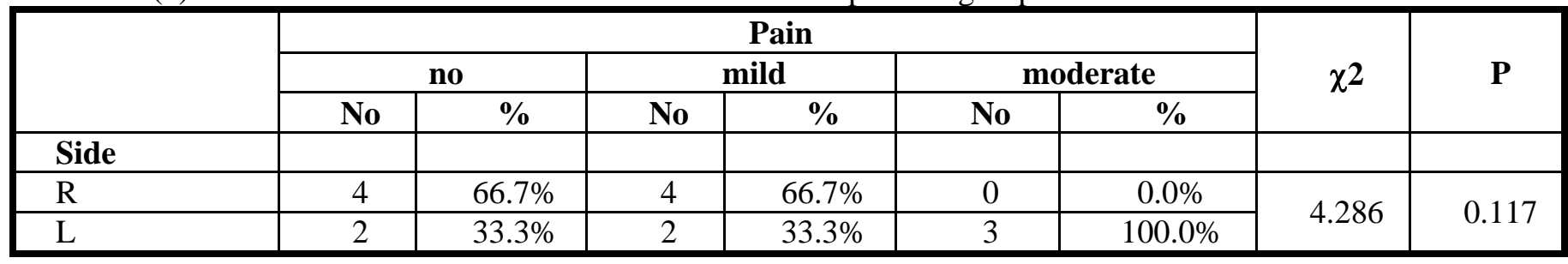

Table (11) show that is no significant relation between fracture side and pain score where $\mathrm{P}$-value $=0.117$.

Table (5): Relation between Pain score and DM in patients group.

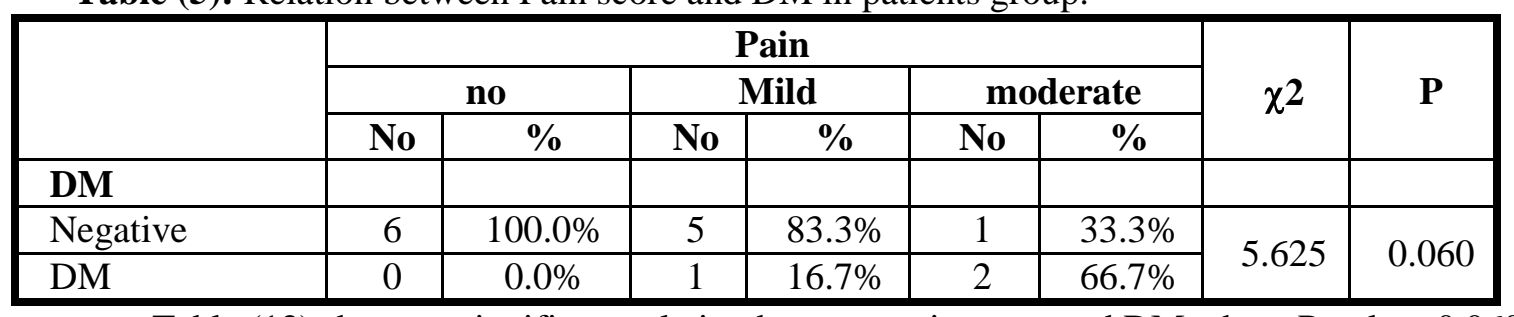

Table (12) show no significant relation between pain score and DM where P-value $=0.060$.

Table (6): Relation between pain score and Age in patients group.

\begin{tabular}{|l|c|c|c|c|}
\hline \multirow{2}{*}{ Mo } & \multicolumn{2}{|c|}{ Age } & \multicolumn{2}{c|}{ ANOVA } \\
\cline { 2 - 3 } & Mean \pm SD & Range & F & P-value \\
\hline Mild & $32.00 \pm 6.63$ & $25-39$ & \multirow{2}{*}{20.502} & $<0.001^{*}$ \\
\hline Moderate & $51.00 \pm 10.94$ & $35-63$ & & \\
\hline & $68.33 \pm 1.53$ & $67-70$ & & \multicolumn{2}{|c|}{ Mild \& Moderate } \\
\cline { 2 - 3 } & No \& Mild & No \& Moderate & \multicolumn{2}{|c|}{$0.012^{*}$} \\
\hline
\end{tabular}

Table (6) show that is significant relation between age and pain score where $\mathrm{P}$-value $=0.012^{*}$.

No pain: the range was (25-39) by Mean \pm SD (32.00 \pm 6.63$)$

Mild: the range was (35-63) by Mean \pm SD $(51.00 \pm 10.94)$

Moderate: the range was (67-70) by Mean \pm SD $(68.33 \pm 1.53)$

Table (7): Relation between pain score and FWD flexion in patients group.

\begin{tabular}{|l|c|c|c|c|}
\hline \multirow{2}{*}{ No } & \multicolumn{2}{|c|}{ Flexion } & \multicolumn{2}{c|}{ ANOVA } \\
\cline { 2 - 3 } & Mean \pm SD & Range & F & P-value \\
\hline Mild & $164.17 \pm 4.92$ & $160-170$ & \multirow{2}{*}{47.461} & $<0.001^{*}$ \\
\hline Moderate & $138.33 \pm 14.72$ & $120-150$ & & \\
\hline & $93.33 \pm 5.77$ & $90-100$ & & \multicolumn{2}{|c|}{ Mild\& Moderate } \\
\cline { 2 - 3 } & No \& Mild & No \& Moderate & \multicolumn{2}{|c|}{$<0.001^{*}$} \\
\hline
\end{tabular}

Table (14) show that is significant decrease relation between FWD flexion and pain score where $\mathrm{P}$-value $=0.001^{*}$.

No pain: the range was $(160-170)$ by Mean \pm SD $(164.17 \pm 4.92)$.

Mild: the range was $(120-150)$ by Mean \pm SD $(138.33 \pm 14.72)$. 
Moderate: the range was $(90-100)$ by Mean \pm SD $(93.33 \pm 5.77)$.

And there was a significant difference between no pain and mild

$\left(\mathrm{P}\right.$-value $\left.=0.001^{*}\right)$ and No pain and moderate $\left(\mathrm{P}\right.$-value $\left.=0.001^{*}\right)$ and significant between mild and moderate $(\mathrm{P}-$ value $=0.001 *)$.

Table (8): Relation between pain score and Abduction in patients group.

\begin{tabular}{|l|c|c|c|c|}
\hline \multirow{2}{*}{ No } & \multicolumn{2}{|c|}{ Abduction } & \multicolumn{2}{c|}{ ANOVA } \\
\cline { 2 - 3 } & Mean \pm SD & Range & F & P-value \\
\hline Mild & $147.17 \pm 8.61$ & $138-160$ & & \\
\hline Moderate & $120.83 \pm 18.28$ & $95-140$ & \multirow{2}{*}{30.732} & $<0.001^{*}$ \\
\hline & $74.33 \pm 4.04$ & $70-78$ & & \\
\cline { 2 - 4 } & No \& Mild & No \& Moderate & \multicolumn{2}{|c|}{ Mild\& Moderate } \\
\hline
\end{tabular}

Table (15) and show that is significant decrease relation between abduction and pain score where Pvalue $=<0.001^{*}$.

No pain: the range was $(138-160)$ by Mean \pm SD $(147.17 \pm 8.61)$.

Mild: the range was $(95-140)$ by Mean \pm SD $(120.83 \pm 18.28)$.

Moderate: the range was (70 -78) by Mean \pm SD (74.33 \pm 4.04$)$.

And there was a significant difference between no pain and mild $\quad\left(\mathrm{P}\right.$-value $\left.=<0.005^{*}\right)$ and No pain and moderate $(\mathrm{P}$-value $=0.001 *)$ and significant between mild and moderate $(\mathrm{P}$-value $=0.001 *)$

Table (9): Relation between pain score and Final constant score in patients group.

\begin{tabular}{|l|c|c|c|c|}
\hline \multirow{2}{*}{ No } & \multicolumn{2}{|c|}{ Const score } & \multicolumn{2}{c|}{ ANOVA } \\
\cline { 2 - 3 } & Mean \pm SD & Range & F & P-value \\
\hline Mild & $84.50 \pm 8.24$ & $76-94$ & \multirow{2}{*}{10.170} & \multirow{2}{*}{$0.003^{*}$} \\
\hline Moderate & $72.00 \pm 10.49$ & $63-90$ & & \\
\hline & $55.33 \pm 8.08$ & $48-64$ & & \multicolumn{2}{c|}{ Mild\& Moderate } \\
\cline { 2 - 4 } & No \& Mild & No \& Moderate & \multicolumn{2}{|c|}{$0.025^{*}$} \\
\hline
\end{tabular}

Table (16) show that is significant decrease relation between Final constant score and pain score where Pvalue $=0.003^{*}$

No pain: the range was (76 -94) by Mean \pm SD (84.50 \pm 8.24$)$.

Mild: the range was (63 -90$)$ by Mean \pm SD $(72.00 \pm 10.49)$.

Moderate: the range was (48 -64) by Mean \pm SD (55.33 \pm 8.08$)$.

And there was a significant difference between no pain and mild $\quad\left(\mathrm{P}-\mathrm{value}=<0.037^{*}\right)$ and No pain and moderate $(\mathrm{P}$-value $=0.001 *)$ and significant between mild and moderate $\left(\mathrm{P}\right.$-value $\left.=0.025^{*}\right)$.

Table (10): Show the Relation between Type of Fracture, age, from inj. to operation, FWD flexion, Abduction, Final constant score.

\begin{tabular}{|l|c|c|c|c|}
\hline \multirow{2}{*}{ Age } & \multicolumn{2}{|c|}{ Parts } & \multirow{2}{*}{ t } & \multirow{2}{*}{ P } \\
\cline { 2 - 3 } & 3 Parts (N=8) & 4 Parts (N=7) & & $0.006^{*}$ \\
\hline Flexion & $37.13 \pm 11.10$ & $58.00 \pm 13.81$ & 3.246 & 0.007 \\
\hline Abduction & $159.38 \pm 10.16$ & $117.14 \pm 25.63$ & 4.087 & $0.004^{*}$ \\
\hline $\begin{array}{l}\text { Contralateral } \\
\text { score }\end{array}$ & $142.88 \pm 12.03$ & $98.29 \pm 26.66$ & 4.077 & $0.003^{*}$ \\
\hline Const score & $89.88 \pm 6.64$ & $82.00 \pm 8.70$ & 1.986 & 0.068 \\
\hline Day operation & $81.13 \pm 10.29$ & $65.14 \pm 13.26$ & 2.627 & $0.021^{*}$ \\
\hline
\end{tabular}

There is significant relation between parts, age, flexion, abduction, const score and day operation but there is no significant relation between parts and contralateral score. 
Table (11): Correlation between Final constant score and Age, from inj. to operation, FWD flexion, Abduction.

\begin{tabular}{|l|c|c|}
\hline \multirow{2}{*}{ Age } & \multicolumn{2}{|c|}{ Const score } \\
\cline { 2 - 3 } & $\mathbf{R}$ & $\mathbf{P}$ \\
\hline Flexion & -0.891 & $<0.001^{*}$ \\
\hline Abduction & 0.855 & $<0.001^{*}$ \\
\hline Contralateral score & 0.885 & $<0.001^{*}$ \\
\hline day operation & 0.911 & $<0.001^{*}$ \\
\hline
\end{tabular}

There was a significant positive correlation between final constant, age, FWD, abduction, contralateral score and day operation.

\section{DISCUSSION}

Fractures of the proximal humerus are increasing due to increased age and resulting older population. Often, these fractures are the result of low speed injuries and complications of osteoporosis and poor overall condition. On the contrary, proximal arm fractures in the younger population usually involve high-speed injury, and fractures are usually complicated with increased interference and soft tissue injury. Although anatomical reduction is not mandatory in the shoulder for normal function, these fractures must be fixed in a stable manner, especially in younger patients to allow early mobilization, rapid recovery and reduced job loss. Surgical options include fractures of three and four parts of the humerus armillary lamination, and techniques of fixation by the skin, transversal wires and hemmiarthroplasty hemorrhage. Traditional internal fixation stabilization methods for proximal humeral fractures include semi-rigid (percutaneous k-wiring, screw fixation, tension band wiring) and rigid (conventional plates and screws, intramedullary nailing) means. Encouraging results with locking plates have made it the implant of choice and has reduced the need for arthroplasty even in difficult four part fractures and in patients with poor bone quality. Several biomechanical studies have validated the efficacy of locking plates in these fractures ${ }^{(5,11-16)}$.

Primary shoulder hemi-arthroplasty should be limited to fractures with articular comminution of the head greater than $40 \%$. Displaced proximal fractures of the humerus, with 3 or 4 fragments according to Neer, present several serious problems for orthopaedic surgeons: complexity of proper reduction; precarious purchase of screws in the spongy bone of the head of the humerus, which has a very low bone stock, especially in postmenopausal women; and risk of necrosis of the head, due to terminal vascularization. In this study, a group of patients with displaced fractures of the proximal humerus, 3 or 4 parts, operated with insertion of PHILOS plates to improve stability ${ }^{(1-5)}$. We analyzed our results and follow up 15 patients for 6 months.
The proximal humeral internal locking system (PHILOS ) is an implant designed for the fixation of proximal humeral fractures and is one of a new generation of locking plates. Its shape is anatomical, conforming to the proximal humerus. In the humeral head component of the plate, locking screws are inserted in multiple directions into the humeral head. In the shaft component of the implant combi-holes provide the option of locking or nonlocking screws to be inserted. Smaller holes allow the passage of sutures or wires to help reattachment of the greater and lesser tuberosities. The implant comes in short and long sizes. The aim of this study was to describe the results of this implant and related complications ${ }^{\left({ }^{(17-20)} \text {. }\right.}$

The introduction of locking plates has brought a new dimension in the treatment of these complex fractures. The presence of multiple angle stable screws in different directions, the availability of sleeves to ease screw insertion and a thin plate profile enable a stable fixation. The conventional deltopectoral approach offers good access to the shoulder joint and is still the approach of choice for fracture fixation among trauma surgeons. Good surgical technique, meticulous handling of fracture fragments and careful preservation of the vascular supply has been shown to produce good long-term results with the deltopectoral approach even in these complex fractures ${ }^{(20)}$.

Development of new-generation (PHILOS Plate) has allowed better results in terms of being more manageable and preserving soft tissues. The plates with mixed holes present biomechanical innovations: while in the conventional plates stability depended on the friction between plate and bone, which is on the anchorage of the screws in the bone, in the PHILOS plates, through the block of the head to screws, the load is transmitted through the implant plate-screw bypassing the bone and working as an internal fixator. This ensures a higher stability and resistance to torsional and axial bending compared to old-generation implants. The PHILOS plates permit a better preservation of the vascularization because the periosteum 
compression is avoided. The advantage, as already evidenced, is that a rigid fixation allows early and effective mobilization. It also depends on correct surgical technique and correct position of the screws in the humeral head. The absence of avascular necrosis of humeral head found in our survey proves the good adaptability and the preservation of the soft tissues guaranteed by the synthesis equipment and the surgical technique adopted ${ }^{(16,19-21)}$.

In open reduction and internal fixation, the goal of the operation is to obtain an anatomic reduction because the rotator cuff needs exact insertion points at the head to guarantee trouble-free function. The second problem of open reduction is exposing the fragments and, at the same time, preserving the soft tissues as much as possible so as not to destroy the connections between the fragments that are important for their blood supply. Finally, an absolutely stable construct must be achieved because external immobilization of the shoulder after an open procedure will lead to stiffness ${ }^{(22)}$.

In this, study 15 patients were treated with 3 and 4 parts fracture, on a prospective study, with follow up for 6 months. The mean age was 46.87 years, ranged from (25-70). The mean constant score of our study is 73.67 range from (48-94). Fazal et al. ${ }^{(23)}$ included 27 patients with two, three and four part fractures. All patients were managed by open reduction and internal fixation by locking plate. Patients were followed up for 6 to 24 (mean, 13) months. The mean final constant score was 70 points (ranged from 28 to 88 points). The score of this study was negatively influenced by one case which developed non-union and avascular necrosis. This may explain why this study had worse results than ours.

In another study conducted by Fankhauser et $\boldsymbol{a l l}^{\left({ }^{(7)}\right.} 28$ patients with two, three and four part fractures were treated by open reduction and internal fixation by locking plate, after one year the average Constant score for all fractures was 74.6 (range, 37-96). For Type A fractures, it was 82.6 (range, 60-96), for Type B it was 78.3 (range, 3795), and for Type $C$ it was 64.6 ,range ( 40-91).

The functional outcome following surgical intervention and insertion of a locking plate was determined using the Constant Score (C.S.). This scoring tool consists of four parameters that are used to assess the function of the shoulder: including pain, daily activity (sleep, work, recreation/sport), range of motion, and strength. The minimum score is 0 and the maximum score is 100 . From analysis of the literature concerning the use of PHILOS plate, our results are considered satisfying results with Constant Scores that range between 48\%-94\%.

Functional recovery has improved up to 6 months after surgery, while, in relation to age, results have worsened progressively with increasing age and also in relation to the compliance with the rehabilitation treatment.

The complication rate in our study is comparable to the literature, where the most recent studies report complication rates between $32-50 \%$ (24). In our study complication rate was $26.66 \%$. No patient developed intraoperative or postoperative vascular injury.

Intraarticular screw perforation was one case (6.6\%) (Case no. 10), this complication may be due to early post-operative fracture collapse. Both implant design and surgical technique may contribute to this. The locking of the screws onto the plate aims to preventing their backing out. Thus if fracture collapse occurs, the screws may penetrate the articular surface, protruding into the glenohumeral joint. This may be more likely to happen if long screws are used with their tips placed very close to the articular surface, or if the articular surface is in-adversely penetrated during drilling. Furthermore, as screws are placed in multiple directions into the humeral head, excessive intraoperative screening is essential to ensure that there is no breaching of the articular surface.one case (6.6\%) (Case no.2) had developed varus collapse conservative treatment and follow up was attempted, full union occurred the patient was satisfied by final outcome so no further management was needed. Two patients suffered from shoulder stiffness and limitation of shoulder movement (13.33\%) for 3.5, 4 months postoperative, and then improved by physiotherapy, (cases no.1, 15). No patient developed hypertrophic keloid or wound dehiscence, no patients had wound complication. No patient developed postoperative serious infection.

In the study that made by Charalambous $\boldsymbol{e t}$ al. ${ }^{(25)}$, the results of the 25 cases, 20 went to union. Five cases $(20 \%)$ required or were considered for revision surgery for non-union or implant failure. Of the 25 implants, 4 had screw protrusion into the gleno-humeral joint (16\%), 4 had screw loosening and backing out (16\%), and 1 plate broke (metal failure) without further trauma (4\%).

The complication rate in the study of Johannes et al. ${ }^{(26)}$ was $19 \%$ (9 of 48 cases). Partial axillary nerve palsy was reported in one case $(2 \%)$. 
In three patients $(6.2 \%)$ cutout of at least one screw through the humeral head into the joint was noted within the first 6 weeks. In four patients (8.3\%) partial AVN developed, which led in all cases to screw perforation of the humeral head, requiring partial or complete implant removal in three patients. Finally, in one (2\%) 91-year-old demented non-compliant patient, the plate loosened during the inpatient stay leading to loss of reduction of the humeral head. In the further course this was managed non-surgically.

On performing the rigid Osteosynthesis of the humerus, extreme care in dissection of the soft tissues and positioning of the plate implant, laterally to the biceps tendon, is recommended, in order to minimize the risk of artery lesion and avascular necrosis. Often, even if the artery is injured, the protection of the soft tissues may preserve posteriormedial vessels and guarantee a certain flow of blood to the humerus head.

The published literature demonstrated a debate regarding the extent of postoperative rehabilitation. Our protocol and recommendation is 3 phases of postoperative physiotherapy.

The first phase of physiotherapy consists of pendulum exercises that started on the first postoperative day and were continued till suture removal. The shoulder was supported in a rightangled sling for 10 days. The passive mobilization was started in the immediate post-operative phase in conformity with the degree of stability of the system and the grade of osteoporosis; in old patients the passive mobilization was postponed for 2 weeks. In the first 2 weeks, single pendulum exercises with passive mobilization up to $30^{\circ}$ were allowed with an increased range of $90^{\circ}$ in the third week. Activeassisted exercises of the shoulder were started at that time and were continued up to 6 weeks. Rotation with passive movements should start after 4 weeks and actively after 6 weeks. Also elbow, wrist, hand exercise were encouraged. In the fourth week, active mobilization without weight was performed and, finally, in the sixth week full active mobilization was allowed.

The second phase of physiotherapy, which was started approximately 6 weeks after surgery, provided the beginning of active exercises: lengthening and progressive isometric strengthening of the rotator cuff and deltoid. The exercises of the third phase generally began after 3 months and characterized by a program of progressive strengthening and maintenance.
In comparison to prosthetic replacement, reduction and Osteosynthesis offer many benefits to the patient; the operation is less invasive, postoperative complications are less and rehabilitation is easier and shorter. Further, the survival time of the prosthetic implant is limited and revision of the shoulder prosthesis is sometimes a difficult procedure.

\section{CONCLUSION}

It could be concluded that locking plates offer more advantages than conventional plates specially when dealing with osteoporotic bone. It is recommended to use locking plate whenever an elderly patient is indicated for internal fixation. Inferomedial comminution of the proximal humerus can lead to failure of locking plate; usage of bone graft is to be considered. Decreasing preoperative lag period is essential to obtain good results. Early passive motion and a well scheduled rehabilitation program have an obvious benefit on the final result.

\section{REFERENCES}

1. Brian LB and Mark M (2008): Fixed-angle Locked Plating of Two-, Three-, and Four-part Proximal Humerus Fractures J Am Acad Orthop Surg., 16:294302

2. Neer CS (1970): Displaced proximal humeral fractures: I. Classification and evaluation. J Bone Joint Surg Am., 52:1077-1089.

3. Neer CS (1970): Displaced proximal humeral fractures: II. Treatment of three-part and four-part displace-ment.J Bone Joint Surg Am., 52: 1090-1103.

4. Naranja RJ and Iannotti JP (2000): Displaced three- and four-part proximal humerus fractures: Evaluation and management. J Am AcadOrthopSurg., 8:373-382.

5. Shane JN, Robert HB, Joseph UB et al. (2007): Innovations in the Management of Displaced Proximal Humerus Fractures J Am AcadOrthopSurg., 15:12-26.

6. Andrew G and Daniel A (2010): Current concepts in evaluation and management of proximal humeral fractures; Current Orthopaedic Practice, 21(5): 435442.

7. Fankhauser F, Boldin C, Schippinger G et al. (2005): A new locking plate for unstable fractures of the proximal humerus. Clinical orthopaedics and related Research, 430:176-81.

8. Strohm PC, Kostler W and Sudkamp NP (2005): Locking plate fixation of proximal humerus fractures. Techniques in Shoulder \& Elbow Surgery, 6:8-13.

9. Charles M, Court-Brown J, Heckman D et al. (2015): Proximal humeral fractures(evaluation), Rockwood and Green's: Fractures in Adults. 
https://otaonline.org/book/952/chapter/53360373/pro ximal-humeral-fractures

10. Bahrs C, Rolauffs B, Südkamp NP et al. (2009): Indications for computed tomography (CT-) diagnostics in proximal humeral fractures: A comparative study of plain radiography and computed tomography. BMC Musculoskelet Disord., 10:33.

11. Hatzidakis AM, Shevlin MJ, Fenton D et al. (2011): Angular-stable locked intramedullary nailing of two part surgical neck fractures of the proximal humerus. A multicenter retrospective observational study. J Bone Joint Surg Am., 93:2172-9.

12. Stedtfeld HW and Mittlmeier T (2007): "Fixation of Proximal Humeral Fractures with an Intramedullary Nail: Tips and Tricks.” Eur J Trauma Emerg Surg., 33(7): 367- 374.

13. Rueger JM, Rücker A, Briem D et al. (2007): "Proximal Humeral. Fractures: Nailing." Eur J Trauma Emerg Surg., 33(7): 357- 366.

14. Park MC, Murthi AM, Roth NS et al. (2003): Twopart and three part fractures of the proximal humerus treated with suture fixation. J Orthop Trauma., 17:319-325.

15. Dimakopoulos P, Panagopoulos A and Kasimatis G (2007): Transosseous suture fixation of proximal humeral fractures. Journal of Bone and Joint Surgery American, 89(8):1700-9.

16. Gerber $C$, Werner $C M$ and Vienne $P$ (2004): Internal fixation of complex fractures of the proximal humerus. J Bone Joint Surg Br., 86:848-855.

17. Greiwe RM and Archdeacon MT (2007): Locking plate technology: current concepts. Journal of Knee Surgery, 20:50-5.
18. Wade RS, Bruce HZ, Jeff OA et al. (2007): Locking Plates: Tips and Tricks. Journal of Bone and Joint Surgery- American, 89: 2298-2307.

19. Haidukewych GJ (2004): Innovations in Locking plate technology. J Am Acad Orthop Surg., 12:205212.

20. Egol KA, Kubiak EN, Fulkerson E et al. (2004): Biomechanics Of locked plates and screws. J Orthop Trauma, 18:488-493.

21. Schütz M and Südkamp NP (2003): Revolution in plate Osteosynthesis: new internal fixator systems. Journal of Orthopaedic Science, 8:252-8.

22. Charles M, Court-Brown J, Heckman D et al. (2015): Proximal humeral fractures(complication), Rockwood and Green's: Fractures in Adults, 37:141219.

23. Fazal MA and Haddad FS (2009): PHILOS plate fixation for displaced proximal humeral fractures. Journal of Orthopaedic Surgery, 17(1):15-18.

24. Röderer G, Johannes E, Markus K et al. (2011): Second generation locked plating of proximal humerus fractures: A prospective multicentre observational study. International Orthopaedics, 35:425-432.

25. Charalambous CP, Siddique I, Valluripalli K et al. (2007): Proximal humeral internal locking system (PHILOS ) for the treatment of proximal humeral fractures. Archives of Orthopaedic Trauma Surgery, 127:205-210.

26. Johannes B, Erhardt G, Roderer K et al. (2009): Early results in the treatment of proximal humeral fractures with a polyaxial locking plate. Archives of Orthopaedic Trauma Surgery, 129:1367-1374. 